\title{
P 070 THE VALUE OF LONGITUDINAL INTERVIEWS IN EXPLORING COPING STRATEGIES OF PATIENTS WITH ADVANCED CANCER AND THEIR CARERS
}

Diane Roberts, ${ }^{1}$ Gunn Grande, ${ }^{1}$ Mari Lloyd Williams, ${ }^{3}$ Lynn Calman, ${ }^{4}$ Lynda Appleton, ${ }^{5}$ Paul Large, ${ }^{1}$ Catherine Walshe ${ }^{2}$. ${ }^{1}$ University of Manchester, Manchester, UK; ${ }^{2}$ Lancaster University, Lancaster, UK; ${ }^{3}$ University of Liverpool, Liverpool, UK; ${ }^{4}$ University of Southampton, Southampton, UK; ${ }^{5}$ The Clatterbridge Cancer Centre NHS Foundation Trust

\subsection{6/bmjspcare-2014-000654.111}

Background Qualitative longitudinal methods, used successfully for research in chronic conditions, can potentially unpick the dynamics of coping with advanced cancer as both a skillset and process. Studies in palliative care commonly suffer from high attrition and raise challenges when exploiting the richness and utility of longitudinal data.

Aims To develop longitudinal methodology by learning from a successful study exploring how and when patients with advanced cancer and their informal carers develop coping strategies.

Methods 54 participants $(n=27$ patients, $n=27$ nominated carers) were recruited to a qualitative longitudinal serial interview study. Using a core topic guide and a semi-narrative, conversational format, two interviews with each participant $(n=86$ interviews) were scheduled 4-12 weeks apart to encompass a range of everyday challenges and life events. A multidimensional approach combined thematic coding and framework analysis to compare patients, carers, dyads and interview points.

Results Researcher sensitivity, responsiveness and planning of second interviews resulted in low attrition between interviews (7.5\%). Interviews were welcomed by participants and their timing was central to understanding how and when participants' developed coping strategies. Participants could reflect in ways which opened up the 'black box' of lived experience and researchers' were able to develop more relevant follow-up questions. This assisted participants to articulate how, why and when they had (or had not) developed particular coping strategies.

Conclusions Supporting the development of coping strategies which benefit patients and carers is both imperative and time critical in palliative care. This study shows that longitudinal qualitative research is valuable when exploring the complex 'everyday realities' of lived experience which are fundamental to quality of life but often develop, unremarked, over time. By integrating opportunities for participant reflection and focusing on 'change over time' this study was able to explore deeper patient-centred insights on which to develop robust evidencebased practice. 